\title{
Identifying and Managing Patients at High Risk for Fractures
}

\section{Conclusions from the Second Spanish Multidisciplinary Forum-Parathyroid Hormone Use in Osteoporotic Patients at High Risk for Fractures}

\author{
Esteban Jódar Gimeno, on behalf of the Forum Meeting Coordinators* and Participants \\ Servicio de Endocrinología y Nutrición Clínica, Hospital Universitario Quirón, Madrid, Spain
}

\begin{abstract}
Purpose: The Second Multidisciplinary Forum: Parathyroid Hormone (PTH) Use in Osteoporotic Patients at High Risk for Fractures (HRF) was conducted to identify specific findings that would be helpful for defining highrisk status and guiding the use of parathyroid hormone 1-84 (PTH1-84) as an anabolic therapy in daily clinical practice. This article summarizes the conclusions from the meeting.

Materials and Methods: Based on three typical case records, and the final conclusions from the first Forum (held in 2010), several questions were posed regarding daily clinical practice definitions of HRF and use of PTH1-84, through a series of 19 meetings throughout Spain. The main discussion topics and agreed conclusions were collected by meeting coordinators and shared at a meeting held in May 2011. After extensive discussions, which also included other organizational and educational matters, some newly agreed conclusions were reached.

Results: The consensus was that an HRF patient is usually thought of as being elderly (aged $>70$ years), with a very low bone mass or a prevalent fracture, and some other associated risk factors. High-risk groups who were identified included patients with neurologic diseases, institutionalized individuals, and patients receiving long-term steroid therapy. PTH1-84 was considered a safe and effective drug, having added value because of its analgesic effect and good level of patient adherence. Opportunities for improved PTH1-84 use were identified, such as better patient selection and follow-up based on localization and specialty. Some improvement opportunities were also detected in organizational and educational areas.

Conclusion: The Forum identified differences between clinical recommendations and daily clinical practice. Some elements, involving both organizational and educational areas that could help to reduce such discrepancies, are described.
\end{abstract}

*A list of the Forum meeting coordinators can be found in the Acknowledgments section. 


\section{Introduction}

In the last 10-20 years, knowledge regarding risk factors and diagnosis of osteoporosis, as well as the various effective therapies that are available, has improved. Taking into account the current deep global economic crisis, responsible use of available limited resources is mandatory.

In such a context, identification of patients with a significant fracture risk is an increasingly important issue, with diverse approaches having been used, based on a combination of several risk factors, morphologic measures, genetic variants, and other inputs. ${ }^{[1-9]}$ While widely disseminated tools to estimate the absolute risk for fractures (e.g. the current FRAX ${ }^{\circledR}$ tool), based on several years' hard work, ${ }^{[10-12]}$ are an undoubtedly useful approach that can be used in daily clinical care where no expertise on osteoporosis is available, a number of limitations remain. ${ }^{[3-5]}$ Moreover, in some countries, only patients with a high risk for fractures according to FRAX $^{\circledR}$ are considered for reimbursement for certain anti-osteoporotic treatments.

Despite several clinical practice guidelines being available for osteoporosis (the Spanish Society for Bone Mineral Research [SEIOMM] guidelines ${ }^{[13]}$ being particularly important in Spain), ${ }^{[13-18]}$ the real use of such guidelines is notoriously low, and their impact on clinical practice is sometimes small. ${ }^{[19,20]}$ Thus, a better understanding of physicians' perceptions and the determinants of real-life clinical practice is required.

To develop this understanding, a project was conducted [the Second Multidisciplinary Forum: Parathyroid Hormone (PTH) Use in Osteoporotic Patients at High Risk for Fractures (HRF)] to expand on the conclusions from the First Multidisciplinary Forum. ${ }^{[21]}$ A strategy was implemented to improve the understanding of factors determining a perceived high risk for osteoporotic fracture and real-life clinical practices associated with the use of anabolic drugs-specifically, parathyroid hormone 1-84 (PTH1-84), which is indicated for high-risk osteoporosis-among a large number of physicians involved in osteoporosis therapy in Spain, the country with the highest use of anabolic therapy in Europe. ${ }^{[22]}$ The project aimed to develop consensus statements that could help guide clinicians in their decision-making processes. The first Forum $^{[20]}$ reached some conclusions on major osteoporosis risk factors and on the identification of patients at the highest risk for fractures, who could benefit from anabolic therapy. Based on these conclusions, two main initial questions were posed for the second Forum:

- What are the characteristics that result in a specific patient being considered an HRF patient in clinical practice, and how can this fact influence treatment selection?

- How is PTH1-84 used in HRF patients? What is the patient profile? When and for how long is PTH1-84 used to treat HRF?

A summary of the conclusions from the second Forum is described here.

This article does not aim to be a systematic review; rather, it aims to provide an account of the discussions that took place at the Forum and conclusions that were reached by physicians in Spain.

\section{Materials and Methods}

The first phase of the second Forum was coordinated by various local leaders and included 19 discussion platforms across Spain, involving more than 300 participants. (The coordinators, institutions, and locations of these Forum meetings are listed in the Acknowledgments section.) All groups used the general report on methods and conclusions from the First Forum and three typical clinical case presentations (table I) to aid discussion on both key questions that were posed. Conclusions were reached by consensus at each meeting and were later shared at a general meeting that was held in Madrid in late May 2011. During this second phase, reports on the final results from the debates among the initial groups were presented by each meeting coordinator. Final conclusions were reached by consensus.

\section{Results}

Taking into account the large number of meetings and participants, including different specialists with different perspectives on osteoporosis, the 
Table I. Clinical case presentations used at the Forum meetings

\begin{tabular}{ll}
\hline Case no. & Characteristic \\
\hline Case 1 & Postmenopausal woman \\
& Low bone mineral density $(-3)$ \\
& Low body mass index \\
& Previous Colles' fracture \\
& Bisphosphonate and calcium supplement therapy \\
& Two vertebral fractures, thoracic-lumbar pain \\
Case 2 & Postmenopausal woman \\
& Low bone mineral density \\
& Family history of hip fracture (in her mother) \\
& Long-term rheumatoid arthritis; corticosteroid therapy \\
& Limited motility, poor quality of life \\
& Postmenopausal woman \\
Case 3 & Low bone mineral density \\
& Family history of fracture \\
& Long-term bisphosphonate therapy \\
& Previous osteoporotic fracture \\
& Current subtrochanteric femur fracture
\end{tabular}

conclusions and reflections are obviously diverse. They have been classified according to the following items for summary and reporting purposes.

\section{The High Risk for Fracture (HRF) Patient Profile}

The HRF patient profile is obviously difficult to define and characterize, as was previously found at a preliminary meeting in 2010 . However, the following features have been identified as the most relevant and/or distinctive (they were all previously identified by the first Forum ${ }^{[20]}$ ):

- Elderly patients: older than 65 years or, as concluded at most meetings, older than 70 years (older than 85 years was even suggested by some groups).

- Prevalent osteoporotic fracture: according to a significant number of meetings, patients with a history of two prevalent osteoporotic fractures (or a single hip fracture) are at particularly high risk. Patients with a single fracture are considered to be potentially high risk if they have additional major risk factors (e.g. frequent falls [more than 3 per year]), are elderly, or have a very low bone mass, among other factors.

- Very low bone mass (T score lower than -3 or -3.5$)$.
- Presence of three or more major risk factors.

- Secondary osteoporosis or primary osteoporosis associated with disease that can result in HRF due to various causes:

- Neurologic diseases, such as cerebrovascular events, Parkinson's disease, spinal cord syndromes, and other disorders that can result in an increased frequency of falls.

- Rheumatologic or other diseases with a risk resulting from the disease itself, and an added risk due to deleterious effects of therapy (for instance, long-term steroid treatment in rheumatoid arthritis patients).

- Institutionalized patients: besides their old age, they usually have vitamin D deficiency, sarcopenia with a low protein intake, a tendency to fall, and several co-morbidities. Both the diseases themselves and their treatment result in HRF.

According to participants at the meetings, when several risk factors are present, the overall risk is substantially increased (for instance, a high-risk patient might be one who is 70 years old with a prevalent vertebral fracture and low femoral bone mass).

Regarding treatment selection, some groups recommended using aminobisphosphonates (alendronate, risedronate, or zoledronate) or strontium ranelate in patients younger than 65 years, with anabolic therapy being a treatment of choice for patients older than 65 years. It must be noted that denosumab, which is now approved for use in this indication, was not available at the time of these discussions.

Use of Parathyroid Hormone 1-84 (PTH1-84) in Clinical Practice

A number of PTHs are available for clinical use. At the Forum meetings, the practical use of PTH184 , a recombinant human PTH, in the treatment of osteoporosis was discussed. As an anabolic therapy, PTH1-84 has shown anti-fracture efficacy in HRF patients, i.e. patients with a prevalent vertebral fracture or very low bone mass. ${ }^{[23]}$ The following conclusions were reached by Forum participants: 
- Anabolic treatment with PTH1-84 is effective, safe, and well tolerated, while adherence to treatment is surprisingly good, considering that it is administered subcutaneously on a daily basis. It has an analgesic effect and results in a substantial improvement in quality of life. According to the perceptions of most participants, it has a more rapid effect than anti-catabolic agents.

- The most relevant patients to receive anabolic therapy with PTH1-84 are:

- Patients recently diagnosed with HRF, i.e. a risk higher than that warranting standard therapy, as mentioned in the previous section.

- Patients receiving anti-catabolic agents (bisphosphonates, selective estrogen-receptor modulators [SERMs], calcitonin) or dualaction drugs (strontium ranelate) and showing poor or no densitometric response (i.e. significant loss of bone mineral density when measured with the same device, and higher than its variability coefficient).

- Patients receiving anti-catabolic or dualaction drugs who present with an osteoporotic fracture, if such a finding seems to be a reasonable indication of therapeutic failure or alters the patient risk profile (for instance, a hip fracture in a patient receiving a drug with no demonstrated efficacy for prevention of such a fracture type [such as some bisphosphonates, SERMs, or calcitonin], or a fracture that should have been prevented with a therapy that has proven efficacy after a reasonable therapy period).

- Patients treated for more than 5-10 years with strong bisphosphonates and showing persistent HRF in spite of such therapy, if a concern exists regarding the potential accumulative effect of such drugs.

- Patients with a significant fracture risk and one of the rare clinical conditions associated with use of strong anti-catabolic drugs, such as jaw osteonecrosis or atypical femur fractures. Although a clearcut cause has not been established, such conditions have been related to an excessive anti-resorptive effect. Thus, use of anabolic agents seems particularly attractive in such cases. Available data on their efficacy are, however, scarce or non-existent.

- Treatment should be started after verification of adequate calcium and vitamin D intake. Anabolic agents, such as PTH1-84, result in new osteoid formation, requiring adequate vitamin D levels to achieve enough mineralization; but some data suggest that most osteoporotic patients are deficient in vitamin D. If vitamin $\mathrm{D}$ cannot be assessed, initiation of average vitamin $\mathrm{D}_{3}$ or $25(\mathrm{OH})$ vitamin $\mathrm{D}$ doses seems a reasonable recommendation before therapy is started. Also, dose equivalents of $800-1000 \mathrm{IU} /$ day should be used during therapy, and increased dietary intake of calcium (up to 1000 or $1200 \mathrm{mg}$ /day) or use of food supplements is recommended.

- Anabolic therapy efficacy has been proven in 18- to 24-month clinical trials; shorter-term use does not guarantee full efficacy. Increased blood or urine calcium levels do not usually cause any clinical manifestations, nor do they require treatment regimen changes. If necessary, calcium and vitamin D supplements should be discontinued and, if this is not sufficient, PTH1-84 should be used every other day. [23]

- Bone mass gains achieved with PTH1-84 anabolic therapy must be consolidated by later administration of anti-catabolic agents. Most available data support later use of aminobisphosphonates, but denosumab may be a good alternative option, based on its anticatabolic strength and reversible effect with no deposit in bone tissue.

- Anabolic agents are currently being used "off label" for some disorders that are not included in their Summaries of Product Characteristics, such as fracture consolidation delay, pseudoarthrosis, after prosthesis implants or total joint replacement, aseptic prosthesis loosening, Südeck's algodystrophy, acute vertebral fractures with poor pain control, or periprosthetic fracture. Despite unproven efficacy in such conditions, therapy is often administered for some months (until clinical resolution of underlying causes), and sometimes for up to 24 months. 


\section{Current Needs and Opportunities for Improvement in Organizational Issues}

Some recommendations were provided regarding the need for improvement in organizational issues, including the following:

- The cost implications of therapy are recognized in a finite-resource scenario, particularly in the present context of a deep economic crisis. Taking into account that available treatments for osteoporosis have proved to be efficient in reducing fracture incidence and complications, available resources should be used in the most efficient way. Thus, such therapies should be used in patients with a significant fracture risk and during life periods when such a risk is really apparent. Use of strong anti-osteoporotic treatments in low-risk patients is unreasonable, whereas therapy denial or failure to recognize disease occurrence in patients at risk is irresponsible.

- A multidisciplinary team approach is recommended for osteoporotic patients; such teams would be particularly effective when treating HRF patients.

- Current interest in osteoporosis is highly variable across medical specialties and geographic areas. No general rule can be established as to which medical specialists are most suitable for the care of osteoporotic patients.

- One situation that needs to be improved is patient care after admission with an osteoporotic fracture; a large number of patients do not receive the correct diagnosis and therapy after initial treatment of the acute event. Such patients show high bone fragility and would mostly benefit from appropriate management.

- At least some members of medical departments currently treating patients with prevalent fractures or HRF patients (orthopedic surgery, rehabilitation, geriatrics, and others) should be involved in protocol development for osteoporotic patient care.

- Primary care physicians should be involved in the diagnosis, treatment, and follow-up of patients initially treated by other specialists (such as orthopedic surgeons). Agreed patient selection processes should be established. There is an obvious need for better information flow across care levels through clinical reports and regular meetings or dedicated multilevel teams.

- Densitometer availability is highly variable. Although densitometry is not mandatory for initial risk assessment, it can be needed in some moderate-risk cases; bone mass may be a critical factor for therapy selection. Thus, public and private health systems should provide such diagnostic tests.

- Clinical inertia is currently limiting best therapy selection, particularly in HRF patients. The patient risk profile should be regularly reassessed, and the efficacy/safety index for a prescribed treatment should be evaluated in order to achieve the best results.

\section{Current Needs and Opportunities for Improvement in Continuing Medical Education}

Continuing medical education needs were also discussed at the meetings. Patients with osteoporosis are currently treated by different medical specialties (primary care physicians, orthopedic surgeons, rheumatologists, rehabilitation specialists, internists, endocrinologists, geriatricians, gynecologists, and others) with highly heterogeneous expertise and involvement in osteoporosis management. High-quality protocols and education programs addressing practical issues associated with managing patients with osteoporosis should be developed. This is particularly true in HRF patients (such as those receiving secondary prevention measures). A general perception of high therapy heterogeneity, not fully supported by patient profile differences, was identified.

Quality of care also seems to show great differences, such as those involving:

- Basic laboratory testing for secondary osteoporosis screening.

- Overall fracture risk assessment.

- Appropriate therapy selection for patients at risk, particularly those receiving secondary prevention measures after an osteoporotic fracture. Clinical practice guidelines based on systematic literature reviews are very useful. Among them, the SEIOMM guidelines, ${ }^{[13]}$ 
which will be updated soon, are probably the most widely accepted guidelines in Spain.

- Regarding PTH1-84 anabolic therapy, some specific needs were recognized. These were the need for regular blood calcium monitoring, a better understanding of its effect (such as increased levels of remodeling markers [including total alkaline phosphatase], potential analgesic effects, improved quality-oflife scores), and improved knowledge of contraindications to its use in patients with a previous cancer history.

- Changes in modifiable risk factors for osteoporosis (smoking habits, excessive alcohol intake, vitamin D deficiency, low calcium intake, and sedentary lifestyle); prevention of falls (correction of visual deficiencies and identification of potential risk behaviors or objects).

- Adequate intake and persistent use of prescribed treatment: prescribing clinicians should provide their patients with appropriate information about how to take drugs and the importance of sustained treatment to achieve full efficacy. General practitioners and family physicians should commonly use effective strategies, such as the Batalla or Morinsky-Green tests, ${ }^{[24]}$ to detect lack of adherence and/or persistence.

- Follow-up needs: a clear-cut difference should be recognized between specific bone metabolism units or services and the usual clinical practice context, which does not require use of densitometry or bone remodeling markers for appropriately selected patients.

- Use of therapy for an adequate period in order to achieve its full efficacy: 24 months for anabolic treatments, or at least 3-5 years for anti-catabolic and mixed treatments.

- Re-assessment or referral of poorly responding patients and patients showing therapy failure to experienced centers.

\section{Consensus Conclusions}

- Patients with a clear-cut prevalent osteoporotic fracture are at HRF (secondary prevention). The risk is higher in patients with multiple fractures. Definition of a high-risk profile, how- ever, is variable across medical specialties because of different clinical risk factors, such as advanced age, long-term steroid use, or neurologic co-morbidities.

- Treatment with PTH1-84 for 18-24 months is safe and effective. It should be used as follows: - First-line therapy for HRF patients.

- Second-line therapy for patients with intolerance of anti-catabolic or mixed therapy, or therapy failure (e.g. fracture occurrence).

- Suspected potential complications due to long-term use of anti-catabolic drugs.

- Individually tailored therapy should be initiated after adequate screening for causes of secondary osteoporosis and correction of modifiable risk factors. Adequate calcium and vitamin D intake should also be ensured.

- Specific strategies are needed to improve patient adherence and persistence, in order to achieve a good outcome.

\section{Discussion}

Relevant medical information about the causes of osteoporosis, the disease course, and therapy has dramatically increased in recent years. Keeping up with such developments is virtually impossible for non-specialists. Thus, carefully evaluated and prioritized clinical practice guidelines, based on systematic review of the available relevant literature and the best international standards, are clearly needed. ${ }^{[25-27]}$ There are multiple clin-

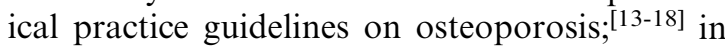
Spain, the SEIOMM guidelines ${ }^{[13]}$ are those most widely accepted.

Such guidelines are, however, scarcely used in daily clinical practice, ${ }^{[19,20]}$ and the situation has not significantly improved in the last few decades. ${ }^{[28]}$ Therefore, better knowledge of characteristics and determinants of real-life clinical practice is clearly needed, to help identify organizational and educational needs in order to minimize the gap between what should be done and what is currently being done in real-life clinical practice.

This is particularly relevant when trying to identify patients with the highest risk for fractures and fracture complications, such as those with functional impairment, loss of health-related 
quality of life, or loss of life years. Taking into account our current need to match available healthcare resources to those patients who are likely to obtain the greatest benefit, an improved understanding concerning the real-life use of one of the most powerful therapies in the current therapeutic armamentarium for osteoporosis is important.

Some limitations are clearly evident in our work, including the lack of a systematic review of the available evidence and the lack of a formal method for discussions. However, our identification of significant differences between recommendations based on systematic reviews developed by scientific societies or various organizations and real clinical practice reflects current perceptions from a large number of physicians involved in real-life osteoporosis care in Spain.

The Forum identified patient selection strategies, treatment rationalization and multidisciplinary team access as focus areas and recommended that changes be made. These could be implemented with minimal cost because they relate to physician behavior and patient management rather than changes to the healthcare infrastructure. The suggestions to improve continuing education programs would require more investment but, given that among Spanish individuals, the tenyear risk for major fracture is $5.5 \%$ for women and $2.8 \%$ for men, ${ }^{[29]}$ the healthcare demands, functional impairment, and quality-of-life consequences represent a considerable burden. Therefore, there is a considerably sized patient population that would benefit from an improvement, and a moderate investment to improve their management would be worthwhile.

Patient selection strategies and therapy selection improvements have been suggested and, most importantly, needs for organizational improvements (such as multidisciplinary team access), and educational requirements that can help design new strategies with an impact on osteoporosis care improvement, have been highlighted.

\section{Acknowledgments}

The author would like to thank Nycomed/Takeda for their assistance in the preparation of the various meetings and, especially, the more than 300 participants at these meetings.
This study was sponsored by Nycomed/Takeda. Medical writing services were provided by Javier Mas of Edmonds SL and funded by Nycomed/Takeda. Native English editing of the manuscript was provided by Andrea Bothwell of inScience Communications, Springer Healthcare, with funding from Nycomed/Takeda. The author, Dr. Esteban Jódar Gimeno, meets the criteria for authorship as recommended by the International Committee of Medical Journal Editors (ICMJE), was fully responsible for all content and editorial decisions, and was involved at all stages of manuscript development. The author declares no conflicts of interest that are directly relevant to the contents of this study.

Coordinators, Institutions, and Locations of the Forum Meetings: Francisco Martinez Martinez, Hospital Virgen de la Arrixaca, Murcia; Javier Aguilar del Rey, Hospital Clinico Malaga, Málaga; Antonio Garcia Sánchez, Hospital San Cecilio, Granada; Vicente Climent, Hospital Xativa, Valencia; Chesus Beltran Audera, Hospital Miguel Servet, Zaragoza; Xoan Miguens, Hospital Monforte de Lemos, León; Ignacio Gonzalez-Busto Mujica, Hospital Central Asturias, Asturias; Santiago Benito Urbina, Hospital San Millán San Pedro, Logroño; Felix Cebeiro, Hospital Basurto, Bilbao; Ernesto Candau, Hospital Rio Hortega, Valladolid; Iñigo Hernandez, Complejo Hospitalario Universitario de Vigo, Vigo; Emilio García Atanes, Hospital Barbanza, Oleiros; Bibiana Villamayor Blanco, Complexo Hospitalario Universitario de Santiago, Santiago; Teresa Torralba, Hospital Cruces, Bilbao; Jose Augusto Lorenzo Rivero, Hospital Dr. Negrin, Gran Canaria; Rosa Gonzalez Crespo, Hospital 12 de Octubre, Madrid; Juan Sanchez Bursón, Hospital Valme, Sevilla; Antonio Sanchez Granados, Hospital Virgen del Rocio, Sevilla; Manuel Roman Torres, Hospital Reina Sofía, Cordoba.

\section{References}

1. Aubry-Rozier B, Lamy O. Fracture risk, new treatments: how does the management of the osteoporosis of elderly change? Rev Med Suisse 2010 Mar 17; 6 (240): 569-70, $572-4$

2. Steiner ML, Fernandes CE, Strufaldi R, et al. Application of Osteorisk to postmenopausal patients with osteoporosis. Sao Paulo Med J 2010 Jan; 128 (1): 24-9

3. Tremollieres FA, Pouilles JM, Drewniak N, et al. Fracture risk prediction using $\mathrm{BMD}$ and clinical risk factors in early postmenopausal women: sensitivity of the WHO FRAX tool. J Bone Miner Res 2010 May; 25 (5): 1002-9

4. Azagra R, Roca G, Encabo G, et al. Prediction of absolute risk of fragility fracture at 10 years in a Spanish population: validation of the WHO FRAX tool in Spain. BMC Musculoskelet Disord 2011 Jan 28; 12: 30

5. Lippuner K, Johansson H, Kanis JA, et al. FRAX assessment of osteoporotic fracture probability in Switzerland. Osteoporos Int 2010 Mar; 21 (3): 381-9

6. LaCroix AZ, Beck TJ, Cauley JA, et al. Hip structural geometry and incidence of hip fracture in postmenopausal women: what does it add to conventional bone mineral density? Osteoporos Int 2010 Jun; 21 (6): 919-29

7. Cheung CL, Sham PC, Chan V, et al. Identification of LTBP2 on chromosome $14 \mathrm{q}$ as a novel candidate gene for bone mineral density variation and fracture risk 
association. J Clin Endocrinol Metab 2008 Nov; 93 (11): 4448-55

8. Blaizot S, Delmas PD, Marchand F, et al. Risk factors for peripheral fractures vary by age in older men-the prospective MINOS study. Osteoporos Int 2011 Jun; 22 (6): 1755-64

9. Lih A, Nandapalan H, Kim M, et al. Targeted intervention reduces refracture rates in patients with incident nonvertebral osteoporotic fractures: a 4-year prospective controlled study. Osteoporos Int 2011 Mar; 22 (3): 849-58

10. Kanis JA, Johnell O, Oden A, et al. Ten year probabilities of osteoporotic fractures according to BMD and diagnostic thresholds. Osteoporos Int 2001 Dec; 12 (12): 989-95

11. Kanis JA, Johnell O, De Laet C, et al. International variations in hip fracture probabilities: implications for risk assessment. J Bone Miner Res 2002 Jul; 17 (7): 1237-44

12. De Laet C, Oden A, Johansson $\mathrm{H}$, et al. The impact of the use of multiple risk indicators for fracture on case-finding strategies: a mathematical approach. Osteoporos Int 2005 Mar; 16 (3): 313-8

13. González Macías J, Guañabens Gay N, Gómez Alonso C, et al. Guías de práctica clínica en la osteoporosis posmenopáusica, glucocorticoidea y del varón. Sociedad Española De Investigación Ósea Y Del Metabolismo Mineral. Rev Clin Esp 2008 May; 208 (Suppl. 1): 1-24

14. Watts NB, Bilezikian JP, Camacho PM, et al. American Association of Clinical Endocrinologists medical guidelines for clinical practice for the diagnosis and treatment of postmenopausal osteoporosis: executive summary of recommendations. Endocr Pract 2010 Nov-Dec; 16 (6): 1016-9

15. Papaioannou A, Morin S, Cheung AM, et al. 2010 clinical practice guidelines for the diagnosis and management of osteoporosis in Canada: summary. CMAJ 2010 Nov 23; 182 (17): 1864-73

16. Compston J, Cooper A, Cooper C, et al. Guidelines for the diagnosis and management of osteoporosis in postmenopausal women and men from the age of 50 years in the UK. Maturitas 2009 Feb 20; 62 (2): 105-8

17. Kanis JA, Burlet N, Cooper C, et al. European guidance for the diagnosis and management of osteoporosis in postmenopausal women. Osteoporos Int 2008 Apr; 19 (4): 399-428

18. Kanis JA, Torgerson D, Cooper C. Comparison of the European and USA practice guidelines for osteoporosis. Trends Endocrinol Metab 2000 Jan-Feb; 11 (1): 28-32

19. Vos E. Osteoporosis guidelines miss big picture. CMAJ 2011 Apr 5; 183 (6): 695
20. Crabtree NJ, Bebbington NA, Chapman DM, et al. Impact of UK national guidelines based on FRAX ${ }^{\circledR}$ — comparison with current clinical practice. Clin Endocrinol (Oxf) 2010 Oct; 73 (4): 452-6

21. Jódar Gimeno E. Conclusiones consensuadas del I Foro Multidisciplinar en el Manejo del Paciente con Alto Riesgo de Fractura (ARF) Osteoporótica. Rev Osteoporos Metab Miner 2010 Jul; 2 (2): 79-86 [online]. Available from URL: http://www.revistadeosteoporosisymetabolismomineral.com/ pdf/articulos/12010020200790086.pdf [Accessed 2012 Nov 15]

22. Hosking D, Alonso CG, Brandi ML. Management of osteoporosis with PTH: treatment and prescription patterns in Europe. Curr Med Res Opin 2009 Jan; 25 (1): 263-70

23. Jódar-Gimeno E. Full length parathyroid hormone (1-84) in the treatment of osteoporosis in postmenopausal women. Clin Interv Aging 2007; 2 (1): 163-74

24. Gil VF, Belda J, Munoz C, et al. Validity of four indirect methods which evaluate therapeutic compliance for arterial hypertension. Rev Clin Esp 1993 Nov; 193 (7): 363-7

25. Appraisal of Guidelines, Research, and Evaluation in Europe (AGREE) Collaborative Group. Guideline development in Europe: an international comparison. Int J Technol Assess Health Care 2000 Autumn; 16 (4): 1039-49

26. AGREE Collaboration. Development and validation of an international appraisal instrument for assessing the quality of clinical practice guidelines: the AGREE project. Qual Saf Health Care 2003 Feb; 12 (1): 18-23

27. Burgers JS, Cluzeau FA, Hanna SE, et al. Characteristics of high-quality guidelines: evaluation of 86 clinical guidelines developed in ten European countries and Canada. Int $\mathbf{J}$ Technol Assess Health Care 2003 Winter; 19 (1): 148-57

28. Cabana MD, Rand CS, Powe NR, et al. Why don't physicians follow clinical practice guidelines? A framework for improvement. JAMA 1999 Oct 20; 282 (15): 1458-65

29. Sanfélix-Genovés J, Sanfélix-Gimeno G, Peiró S, et al Prevalence of osteoporotic fracture risk factors and antiosteoporotic treatments in the Valencia region, Spain. The baseline characteristics of the ESOSVAL cohort. Osteoporos Int. Epub 2012 May 23

Correspondence: Dr. Esteban Jódar Gimeno, Servicio de Endocrinología y Nutrición Clínica, Hospital Universitario Quiron Madrid, Diego Velazquez 2, 28223 Pozuelo de Alarcón, Madrid, Spain.

E-mail: ejodar.mad@quiron.es 